\title{
Accretion-powered chromospheres in classical T Tauri stars ${ }^{\star}$
}

\author{
P. P. Petrov ${ }^{1}$, G. F. Gahm ${ }^{2}$, H. C. Stempels ${ }^{3}$, F. M. Walter ${ }^{4}$, and S. A. Artemenko ${ }^{1}$ \\ ${ }^{1}$ Crimean Astrophysical Observatory, p/o Nauchny, 98409 Crimea, Ukraine \\ e-mail: petrov@crao.crimea.ua \\ 2 Stockholm Observatory, AlbaNova University Centre, Stockholm University, 10691 Stockholm, Sweden \\ 3 Department of Physics and Astronomy, Uppsala University, Box 516, 75120 Uppsala, Sweden \\ 4 Department of Physics and Astronomy, Stony Brook University, Stony Brook, NY 11794-3800, USA
}

Received 15 February 2011 / Accepted 17 August 2011

\begin{abstract}
Context. Optical spectra of classical T Tauri stars (cTTS) are rich in emission lines of low-excitation species that are composed of narrow and broad components, which indicates the existence of two emitting regions with different kinematics, densities, and temperatures. The photospheric spectrum is often veiled by an excess continuous emission. This veiling is usually attributed to radiation from a heated region beneath the accretion shock. The broad emission lines of $\mathrm{H}_{\mathrm{I}}, \mathrm{He}$ II, Ca II, Fe II, and other species are thought to form in a larger volume of gas.

Aims. The aim of this research is to clarify the nature of the veiling, and whether the narrow chromospheric lines of $\mathrm{Fe}_{\mathrm{I}}$ and other metals represent a standard chromosphere of a late-type star, or are induced by mass accretion.

Methods. We carried out high-resolution spectroscopy of selected cTTS with a special focus on DR Tauri and followed variations of chromospheric features, such as narrow Fe I emission lines, and accretion signatures such as the veiling continuum and the He II line emission.

Results. We found that the amount of veiling in DR Tau varies from practically nothing to factors more than 10 times the stellar continuum intensity, and that the veiling is caused by both a non-photospheric continuum and chromospheric line emission filling in the photospheric absorption lines. The latter causes differential veiling because stronger lines are more veiled. We developed methods to separate the two sources of veiling. Several veiled T Tauri stars show a common effect: the radial velocities of photospheric and chromospheric lines vary in anti-phase. This is caused by an area with enhanced chromospheric emission, which is offset from the pole of rotation and is associated with the hot spot formed at the footprint of the magnetic funnel of mass accretion.

Conclusions. The enhanced chromospheric emission in cTTS is linked not only to solar-like magnetic activity, but is powered to a greater extent by the accreting gas. We suggest that the area of enhanced chromospheric emission is induced by mass accretion, which modifies the local structure of stellar atmosphere in an area that is more extended than the hot accretion spot. The narrow emission lines from this extended area are responsible for the extra component in the veiling through line-filling of photospheric absorption lines.
\end{abstract}

Key words. stars: variables: T Tauri, Herbig Ae/Be - stars: individual: DR Tau - stars: individual: S CrA - stars: individual: RW Aur stars: individual: RU Lup - stars: individual: DI Cep

\section{Introduction}

The T Tauri stars are pre-main sequence stars of low mass. Spectroscopically they are characterized by emission lines superimposed on a photospheric spectrum of late type. In the classical T Tauri stars (cTTS) the emission line spectrum is very rich and related to magnetospheric accretion from dusty disks. The T Tauri stars rotate faster than most late-type main-sequence stars, and the enhanced emission in the weak-line stars, those not exhibiting evidence of accretion, is adequately explained in terms of enhanced solar-like magnetic activity. The same dynamo effect should be present in the cTTS as well, but in addition enhanced line emission is generated in the accreting gas, which flows from the circumstellar disk to the star, where it eventually heats up and releases energy in strong shocks at the stellar surface. Accordingly, the emission lines are much broader in classical $\mathrm{T}$ Tauri stars than expected from chromospheric emission

\footnotetext{
* Based on observations collected at the Nordic Optical Telescope, La Palma in Spain (runs 34-011 and 40-006) and the European Southern Observatory in Chile (run 075.C-0292).
}

alone. For reviews of properties and models of cTTS see Petrov (2003) and Bouvier et al. (2007).

In cTTS the photospheric absorption lines are weakened by what is commonly attributed to an overlying continuous excess emission. The energy distribution of this veiling has been matched with black body radiation at a temperature of $\sim 10^{4} \mathrm{~K}$, or alternatively with Paschen continuous emission (Basri \& Batalha 1990; Hartigan et al. 1991, 1995; Valenti et al. 1993; Calvet \& Gullbring 1998; Gullbring et al. 1998). The veiling is attributed to radiation from "hot spots", small areas (sometimes rings) of heated gas at the footprints of the accretion flows. $\mathrm{T}$ Tauri stars are photometrically variable, both from rotational modulation of surface features and stochastic events (e.g., flares, changes in mass accretion rates). The changes in line intensities and profiles in many cTTS are commonly attributed to variations in the accretion flow. The veiling levels can vary dramatically, and in the most active cTTS veiling may change from a tiny fraction of the intensity of the photospheric continuum to factors of several times this level. The degree of veiling is commonly used to estimate accretion rates in CTTS. 
Intuitively, one would expect that when the veiling changes, the stellar brightness would change accordingly. However, there had been several hints that this is not always the case. Gahm et al. (2008) demonstrated that in four cTTS with very pronounced emission line spectra and strong irregular light variations, the veiling variations clearly did not follow this pattern. Substantial changes in the veiling were accompanied by only minor changes in $V$ and $B$, which in combination with an analysis of variations in equivalent widths of emission lines led the authors to conclude that veiling is not solely related to an excess continuum emission. Furthermore, it was demonstrated that the photospheric absorption lines are weakened due to filling-in by narrow, emission line components. While the above shows that the veiling effect is caused by at least two different processes, it is still unclear which mechanism causes the appearance of these narrow emission features.

In order to investigate the origin of the narrow-lined chromospheric emission features, we performed a detailed investigation of the behavior of veiling in one of the most variable cTTS, namely DR Tauri. Our observations show this star to vary from a state of practically no veiling to levels of more than 10 times the continuum flux. At high veiling levels narrow emission features are clearly present in this object. In addition we find periodic variations in radial velocity of both absorption lines as well as of the narrow emission components, which, as we will show in this paper, provides indications on their origin.

DR Tau is an unusually "active" cTTS, in the sense that the emission line spectrum is exceedingly rich and variable, and that the photometric variations are large and primarily related to physical changes in the plasma surrounding the star. There are many other well-known cTTS with similar properties, for which similar conclusions can be drawn regarding the nature of the veiling and the radial velocity fluctuations. We will illustrate this in the present paper by complementing our analysis with stars that show similar spectral line variations, namely RW Aur A, DI Cep, RU Lup, and S CrA SE.

DR Tau was for a long period a relatively faint star until in 1976 it rather suddenly brightened by several magnitudes in the blue (Chavarria-K. 1979), and the star is sometimes classified as an EXor star (see e.g. Lorenzetti et al. 2009). However, since that time it has stayed at this bright level but with dramatic and fairly irregular light variations (Artemenko et al. 2010). Tentative photometric periods proposed by Richter et al. (1992), Bouvier et al. (1993, 1995) differ by several days, and are different from a more recent determination by Percy et al. (2010), who assign a period of 5.0 days. This is also in the same range of possible periods found from near-infrared (NIR) photometry by Kenyon et al. (1994), who relate the periodicity of DR Tau to a hot spot on a rotating star with a magnetic axis inclined to the stellar rotation axis.

Pronounced spectral variability in DR Tau was recognized early (Bertout et al. 1977; Krautter \& Bastian 1980; Mundt 1984; Aiad et al. 1984; Appenzeller et al. 1988). The strong emission lines on occasion show inverse P Cygni structures, which appear and disappear on time-scales as short as a few hours (Smith et al. 1997, 1999). Basri \& Batalha (1990) demonstrated that the veiling is large and variable, and subsequent investigations of relations between variations in emission lines and veiling led Guenther \& Hessman (1993) and Hessman \& Guenther (1997) to conclude that in principle the observations were consistent with the concept of magnetospheric accretion, with gas flowing along dipole magnetic fields to a hot region on the visible surface. However, the data are inconsistent with constant accretion in a simple magnetic dipole, because irregular mass accretion and possible flare-line events distort the Balmer line profiles. In their long-term study of spectral variablity in DR Tau, Alencar et al. (2001) were not able to recover any periodicity in the spectral changes. However, Hessman \& Guenther (1997) found evidence of a periodicity of 4.5-5 days in the equivalent widths of strong lines, and Johns \& Basri (1995) reported a 5.1 day period in components in $\mathrm{H} \alpha$. Because these spectral periods are similar to some of the photometric periods presented above, one may suspect that both phenomena are related to rotational modulation, and that the period of rotation of DR Tau is in the range of 4.5-5 days.

The complex emission line profiles and their dramatic changes can be better understood if the profiles are decomposed into different components arising from different emitting volumes around the star. The accreting gas produces Balmer line emission, sometimes with inverse P Cygni absorption components, and the broad metallic lines reflect the bulk motion of this in-falling gas (see e.g. Muzerolle et al. 1998a,b). Normal P Cygni absorption components are seen as well, indicating outflow in the form of massive winds or jets. The P Cygni absorptions are particularly distinct in some far-ultraviolet lines, like the Mg II doublet (Kravtsova \& Lamzin 1996; Ardila et al. 2002). The He I lines, particularly the NIR line at $10830 \AA$, display absorption both from the in-falling and out-flowing gas as analysed in a series of papers by Beristain et al. (2001), Edwards et al. (2003, 2006), Kwan et al. (2007) and Fischer et al. (2008). In all, DR Tau stands out as a cTTS with an unusually dynamic and very complex interplay between accreting and ejected gas.

As in many cTTS, the He lines in DR Tau display a narrow emission component, which normally is attributed to postshock gas at the footprints of the accretion flows (Lamzin et al. 1996; Beristain et al. 1998). However, in DR Tau similar narrow components with a full-width half-maximum (FWHM) of $\sim 20 \mathrm{~km} \mathrm{~s}^{-1}$ were also found in lines of Ca II, Fe I and Fe II (Batalha et al. 1996; Beristain et al. 1998). In fact, Batalha et al. found a positive correlation between line strength and veiling and speculated that the narrow component is related to a heated stellar atmosphere close to the accretion zone a "hot chromosphere".

Below we present our observations and reduction methods in Sect. 2. The methods of analysis and results are given in Sect. 3 and are discussed in more detail Sect. 4. Section 5 summarizes our results.

\section{Observations and data reductions}

Observations of DR Tau were carried out during two runs in 2007 and 2009/2010 at the Nordic Optical Telescope (NOT) with the fiber-fed echelle spectrograph FIES (Frandsen \& Lindberg 2000). The data were processed with FIEStool ${ }^{1}$. This software package is fine-tuned for reducing data obtained with the FIES spectrograph and performs the full set of echelle spectral reduction steps, including bias subtraction, modelling of scattered light, extraction through two-dimensional modelling of the spectral orders, flat-fielding and wavelength calibration.

The extracted spectra have a resolution of $\lambda / \Delta \lambda \approx 47000$ and cover a wavelength range of 3650-7250 $⿱$. In Table 1 we summarize observation dates, the number of 20-min exposures obtained in each night and signal-to-noise ratio per pixel in the continuum of nightly averaged spectra. The last two columns give the mean veiling factors at 6110-6160 $\AA$ and their standard

\footnotetext{
1 http://www.not.iac.es/instruments/fies/fiestool/ FIEStool.html
} 
Table 1. DR Tau observations.

\begin{tabular}{cccccc}
\hline \hline Year & HJD 245... & $N$ exp & $S / N$ ratio & $\mathrm{VF}_{6110}$ & $\sigma_{\mathrm{VF}}$ \\
\hline 2007 & 4101.4056 & 2 & 65 & 4.4 & 0.5 \\
& 4103.5857 & 2 & 45 & 1.5 & 0.3 \\
& 4104.4278 & 2 & 55 & 1.2 & 0.3 \\
& 4105.6235 & 2 & 80 & 7.5 & 1.0 \\
2009 & 5192.5623 & 4 & 110 & 4.8 & 0.5 \\
& 5193.4952 & 3 & 80 & 7.0 & 1.0 \\
& 5194.4984 & 3 & 60 & 5.5 & 0.5 \\
& 5195.4934 & 4 & 90 & 3.8 & 0.5 \\
& 5196.4748 & 4 & 70 & 4.6 & 1.0 \\
2010 & 5197.5002 & 4 & 150 & 7.0 & 0.7 \\
& 5198.5539 & 4 & 90 & 4.6 & 0.5 \\
& 5199.5762 & 2 & 35 & 5.0 & 1.5 \\
& 5201.4676 & 2 & 60 & 3.6 & 0.5 \\
\hline
\end{tabular}

Notes. See the main text for a more detailed description of the columns.

deviations (see Sect. 3.3.1). In addition, we obtained spectra of the K7V flare star HD $28343\left(\mathrm{BD}+21^{\circ}\right.$ 652) for comparison purposes.

This study focuses on DR Tau, but includes also supporting data from the two components of S CrA (Gahm et al. 2008), RU Lup (Stempels et al. 2007), RW Aur A (Petrov et al. 2001), and DI Cep (Gameiro et al. 2006).

\section{Data analysis and results}

We start with a brief description of the spectra of DR Tau and the determination of the basic stellar parameters: temperature, gravity, radius, and rotation, and continue with an analysis of the veiling as a tracer of accretion, and radial velocities of narrow emission lines as indicators of chromospheric surface inhomogeneities.

\subsection{General spectral properties}

We present in Fig. 1 two typical segments of the DR Tau spectrum. Here, we averaged all 13 spectra to increase the signalto-noise ratio. For comparison we also show a spectrum of the template K7 star artificially veiled by a factor of four, so that the strength of the photospheric lines become similar to those of DR Tau.

The main features in the spectrum of DR Tau are as described by Beristain et al. (1998). The strongest emission is in the hydrogen Balmer lines and $\mathrm{Ca}$ II $\mathrm{H}$ and $\mathrm{K}$. Weaker emission is seen in neutral and singly ionised permitted lines of $\mathrm{Fe}$ I, Fe II, He I, He II, Ti II, Na I and $\mathrm{Ca}$ I, and in forbidden [O I], [S II], and [N II].

The weakest emission lines are narrow $(F W H M \sim$ $20 \mathrm{~km} \mathrm{~s}^{-1}$ ), while stronger emission lines of metals exhibit a two-component structure: a similar narrow component as well as a broad component with $F W H M \sim 100 \mathrm{~km} \mathrm{~s}^{-1}$. The broad component gradually grows stronger as the emission lines themselves become stronger, as is evident from the panels shown in Fig. 1. The stronger He I and Fe II lines (for example Fe II $\lambda 4924$ and $\lambda 5018 \AA$ ) show additional red-shifted absorption extending to $+350 \mathrm{~km} \mathrm{~s}^{-1}$. These signatures of infall vary with time and are also present in the higher Balmer lines as shown in Fig. 2. The profiles of $\mathrm{H} \alpha$ and other Balmer lines are split by deep broad absorption, centred at $-100 \mathrm{~km} \mathrm{~s}^{-1}$, which indicates an outflow. Typical Balmer line profiles can be found in Alencar et al. (2001), where the Balmer emission was analysed in greater depth.
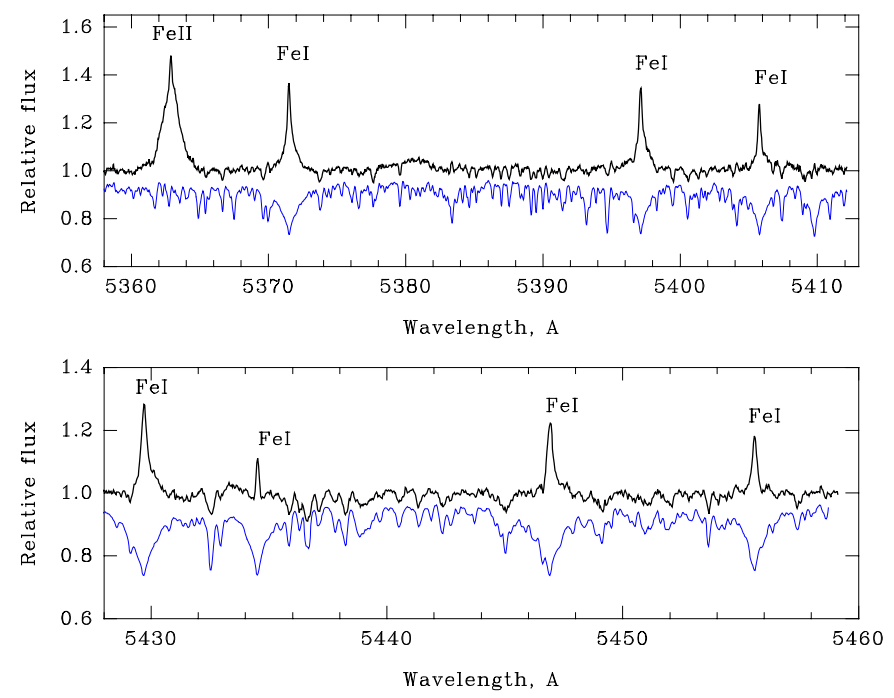

Fig. 1. Segments of the averaged spectrum of DR Tau (solid line) as compared to the K7 V template HD 28343 (thin line) veiled artificially by a factor of four. In this and other figures the wavelength scale is in the stellar rest frame.
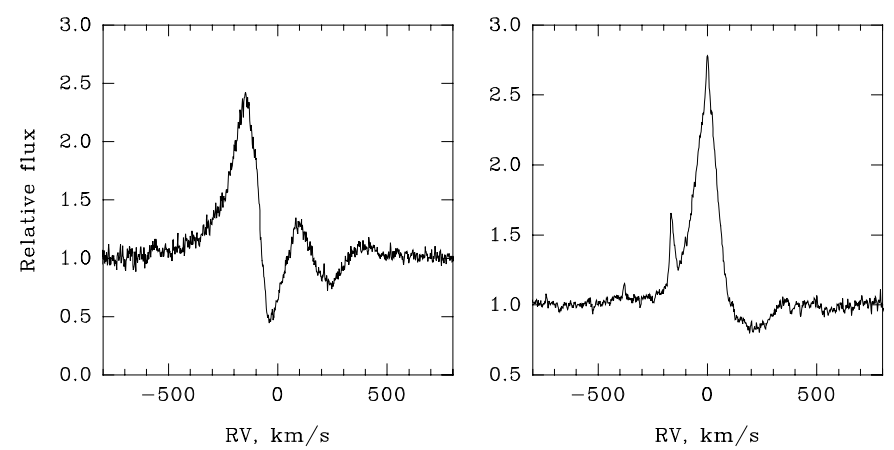

Fig. 2. Emission line profiles with red-shifted absorption components in the spectrum of DR Tau on HJD 2455 192. Left: H $\delta$, right: Fe II $5018.43 \AA$ (the narrow emission peak on the blue wing is He I $5015.67 \AA$ ).

Photospheric lines are detectable longward of $\sim 4500 \AA$ in the averaged spectrum, while during some nights with higher veiling and/or lower signal-to-noise ratio many lines are not detectable. Nevertheless, in some spectral intervals the photospheric lines are always visible, which makes it possible to follow night-tonight variations of the veiling. In moments of high veiling some photospheric lines (e.g. Fe I, multiplet 15) turn from absorption into pure emission.

\subsection{Stellar parameters}

Spectral types assigned previously for DR Tau fall in a fairly narrow range, from K5 to M0. The variable and often high veiling of the photospheric absorption lines makes it difficult to derive precise stellar parameters. However, we did obtain one spectrum of DR Tau in a relatively calm state $(\mathrm{HJD}=2454104)$, which we will refer to as the low-veiling spectrum.

This low-veiling spectrum was compared to a grid of synthetic spectra calculated for $T_{\text {eff }}=3500,3750,4000,4250$, and $4500 \mathrm{~K}$ with $\log g$ values of 3.0, 3.5, and 4.0. This grid was calculated using the code by Berdyugina (1991) and Kurucz' models. Atomic line data were retrieved from the VALD database (Kupka et al. 2000). The best fit was found for 


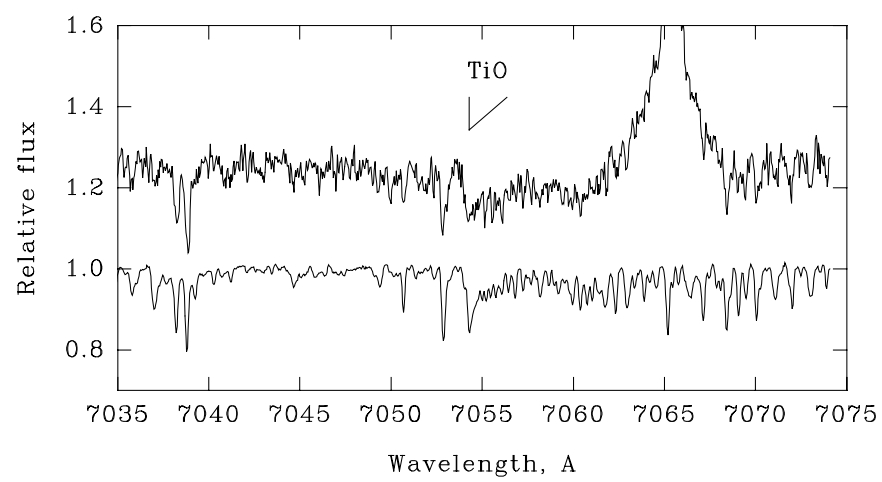

Fig. 3. Molecular TiO band at $7054 \AA$ A Upper: the low-veiling spectrum of DR Tau. Lower: the spectrum of HD 28343, (K7 V), artificially veiled by a factor of 1.0 .

$T_{\text {eff }}=4000-4250 \mathrm{~K}$ and $\log g=3.5-4.0$. In order to check the spectral type with molecular features, we used the spectrum of HD 28343 (spectral type K7V) along with M0V and K5V spectra collected earlier with FIES. The spectrum of DR Tau is best matched with the K7V spectrum, as is illustrated in Fig. 3, where the template spectrum is artificially veiled by a factor of 1.0 . The TiO bandhead at $7054 \AA$ is also consistent with a spectral type of $\mathrm{K} 7 \mathrm{~V}$ for DR Tau, and we adopt $T_{\text {eff }}=4100 \pm 200 \mathrm{~K}$ and $\log g=$ $3.7 \pm 0.5$ for the photosphere of DR Tau.

In Fig. 4 we compare the low-veiling spectrum of DR Tau with a synthetic spectrum in the region of Ca I $\lambda 6124 \AA$. The synthetic spectrum was calculated for $T_{\text {eff }}=4100 \mathrm{~K}, \log g=3.7$, $v_{\text {mic }}=1.5 \mathrm{~km} \mathrm{~s}^{-1}, v_{\text {mac }}=1.5 \mathrm{~km} \mathrm{~s}^{-1}$ and $v \sin i=5 \mathrm{~km} \mathrm{~s}^{-1}$, convolved with the instrumental profile of FIES and veiled by factor of 1.3. The profiles of the VI, Ti I and Ba II lines fit fairly well. A $\log g$ in the range 3.5-4.0 gives a reasonably good fit to the broad wings of the $\mathrm{Ca}$ I line. The lines of $\mathrm{Fe}_{\mathrm{I}}$ are partly filled in with chromospheric emission (see below).

The projected rotational velocity $v \sin i=5 \pm 1 \mathrm{~km} \mathrm{~s}^{-1}$ was found from the widths of many metal lines as fitted with the synthetic spectrum in the region 5580-6150 $\AA$. Note that $v \sin i$ is somewhat less than the $F W H M$ of the instrumental profile $\left(\approx 6.5 \mathrm{~km} \mathrm{~s}^{-1}\right)$. Nevertheless, the high signal-to-noise ratio in our spectra enables us to detect this low value of the rotational broadening of photospheric lines.

Assuming that at minimum brightness the photosphere is less affected by accretion, we can estimate the stellar luminosity and radius. From an extended set of photometric data from 1986-2003 (Grankin et al. 2007), $V_{\min }=13.0 \pm 0.30$ and the corresponding colour $V-R=1{ }^{\mathrm{m}} 40 \pm 0.08$, which gives $A_{V}=$ $0.93 \pm 0.30$. From the corresponding minimum $B-V$ colours we find a faintest $B$ magnitude that is similar to what DR Tau had in the first half of the 20th century (Chavarria-K. 1979). Hence, there is no indication that the stellar parameters have changed following the general increase in brightness around 1976. The circumstellar component of the extinction is probably small because the star is seen close to pole-on (see also the discussion in Sect. 3.3.2). With a distance to DR Tau of $d=140 \mathrm{pc}$ (e.g. Bertout \& Genova 2006) we obtain the following stellar parameters: $L=0.52 \pm 0.15 L_{\odot}$ and $R=1.46 \pm 0.20 R_{\odot}$. Earlier published estimates of the radius range from $1.2 R_{\odot}$ (Bertout $\&$ Basri 1988) to $2.7 R_{\odot}$ (Johns-Krull \& Gafford 2002).

Regarding other programme stars, the primary and secondary of S CrA have earlier been classified as G5 Ve and K5 Ve, respectively (Carmona et al. 2007). However, our longslit UVES/VLT spectra yield very similar parameters for the two

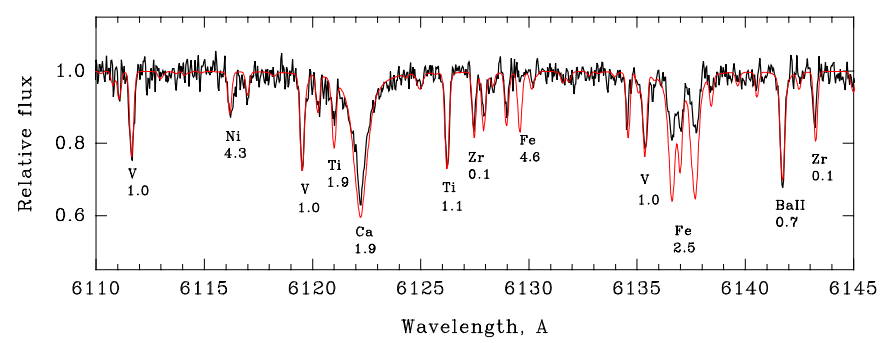

Fig. 4. Solid line: the low-veiling spectrum of DR Tau. Thin line: synthetic spectrum for $T_{\text {eff }}=4100 \mathrm{~K}, \log g=3.7$ and $v \sin i=5 \mathrm{~km} \mathrm{~s}^{-1}$, convolved with the instrumental profile and veiled by a factor of 1.3. Elements and lower level excitation potentials $(\mathrm{eV})$ are indicated.

components, namely $T_{\text {eff }}=4250 \pm 150 \mathrm{~K}, \log g=4.0 \pm 0.2$ and $v \sin i=12 \pm 1 \mathrm{~km} \mathrm{~s}^{-1}$. Furthermore, RW Aur A has a spectral type of $\mathrm{K} 1-\mathrm{K} 4, v \sin i \approx 20 \mathrm{~km} \mathrm{~s}^{-1}$ (Petrov et al. 2001); RU Lup has $T_{\text {eff }}=3950 \pm 200 \mathrm{~K}, \log g=3.9 \pm 0.3$, and $v \sin i=9.0 \pm$ $0.9 \mathrm{~km} \mathrm{~s}^{-1}$ (Stempels \& Piskunov 2002), and DI Cep has spectral type G8 and $v \sin i=20.0 \pm 1.5 \mathrm{~km} \mathrm{~s}^{-1}$ (Gameiro et al. 2006).

\subsection{Two sources of veiling}

As discussed early by e.g. Bertout (1984), the photospheric spectrum of a cTTS can be veiled by 1) an additional nonphotospheric excess continuum and 2) line emission filling in photospheric absorption lines.

\subsubsection{Traditional veiling measurements}

Traditionally, the veiling factor (VF) is determined from the equivalent width $(E W)$ of a photospheric line measured in the spectrum of DR Tau and in a template (e.g., synthetic) spectrum:

$\mathrm{VF}=E W_{\text {synt }} / E W_{\mathrm{DR}}-1$.

From measurements of numerous photospheric absorptions in the average spectrum of DR Tau no significant correlation was found either in veiling versus wavelength, or in veiling versus excitation potential of different lines, within the wavelength range 4500-6800 $\AA$ and excitation potential range $0-5 \mathrm{eV}$. Although it is commonly accepted that veiling increases towards the blue, the wavelength dependence is not always well defined because of the large scatter in the veiling factors derived from different spectral lines (e.g. Basri \& Batalha 1990). In Fig. 5 we show the ratio of $E W \mathrm{~s}$ as measured in the spectra of the K7V template and DR Tau as a function of wavelength. Each symbol represents one spectral line. Note that the large scatter of points is caused not only by errors of measurements of $E W \mathrm{~s}$, but also by the dependence of veiling on line strength (see below).

Not all absorption lines that are visible in the averaged spectrum can be detected in individual spectra. We therefore carefully selected spectral regions where photospheric lines are clearly visible in all spectra of DR Tau, even in those with high veiling, and which never turn into emission: 5580-5605 (six lines of CaI), 6000-6040 $\AA$ (four lines of MnI and $\mathrm{Fe}_{\mathrm{I}}$ ), 6110-6160 $\AA$ (five lines of $\mathrm{V}_{\mathrm{I}}, \mathrm{Ti} \mathrm{I}, \mathrm{Ba}$ II, NaI) and 6460-6480 $\AA$ (two lines of Ca I). In these regions we measured the corresponding values of the VF in each spectrum of DR Tau using the K7V template. Figure 6 shows the night-to-night variations of the veiling. The VF at $6110-6160 \AA$ and its standard deviation are also given in the last two columns in Table 1. 


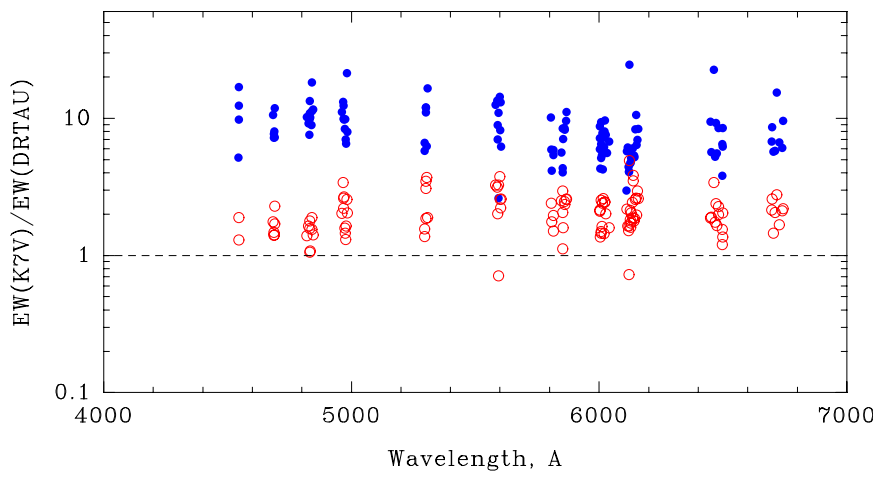

Fig. 5. Ratio of $E W \mathrm{~s}$ in the K7V template and DR Tau. Open circles: low-veiling spectrum, filled circles: average spectrum. The horizontal dashed line marks the case of zero veiling.
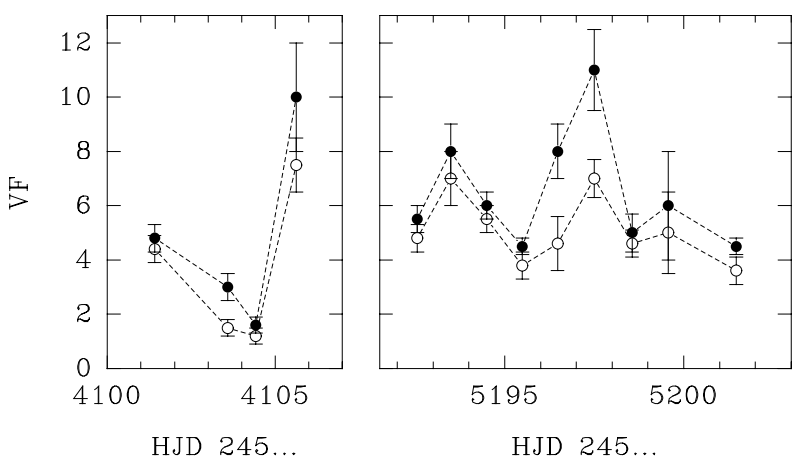

Fig. 6. Night-to-night variations of the veiling factor in DR Tau. Filled circles: spectral region 6000-6040 $\AA$ (lines of Mn I and Fe I). Open circles: spectral region 6110-6160 A (lines of V I, Ti I, Ba II, Na I).

\subsubsection{Distinguising the excess continuum from chromospheric line emission}

Unfortunately, the veiling measurements we perform above cannot discriminate between the effect of a veiling continuum and the effect of chromospheric line emission filling-in the photospheric lines. We therefore used the following three methods to separate and quantify these two effects.

The first method is based on measurements of the forbidden lines and is similar to the method used by Gahm et al. (2008). Forbidden emission lines of [O I] at 5777.3, 6300.2 and $6363.8 \AA$ and $\left[\mathrm{S}_{\mathrm{II}}\right]$ at 6717.0 and $6731.3 \AA$ are usually present in spectra of cTTS. These forbidden lines are formed in a large volume extending far away from the star, as compared to permitted emission lines, and can therefore be expected to be intrinsically stable on a time scale of days. Hence, the observed night-to-night variations of $E W \mathrm{~s}$ of the forbidden lines are mainly caused by continuum variations.

Figure 7 shows that there is a strong correlation between $E W \mathrm{~s}$ of photospheric absorption lines and forbidden lines of [OI]. The existence of this correlation indicates that both the photospheric and the [OI] lines are affected by the continuous veiling component. However, the range of variability for the photospheric lines is twice as large as for the [O I] lines: the $E W \mathrm{~s}$ of [OI] lines change by a factor of 2.7 , while the $E W$ s of photospheric lines change by a factor of of 5.3. This means that another source of veiling contributes as much as the continuum veiling component.

The second method is based on the effect of differential veiling. Here, we measured from the average spectrum of

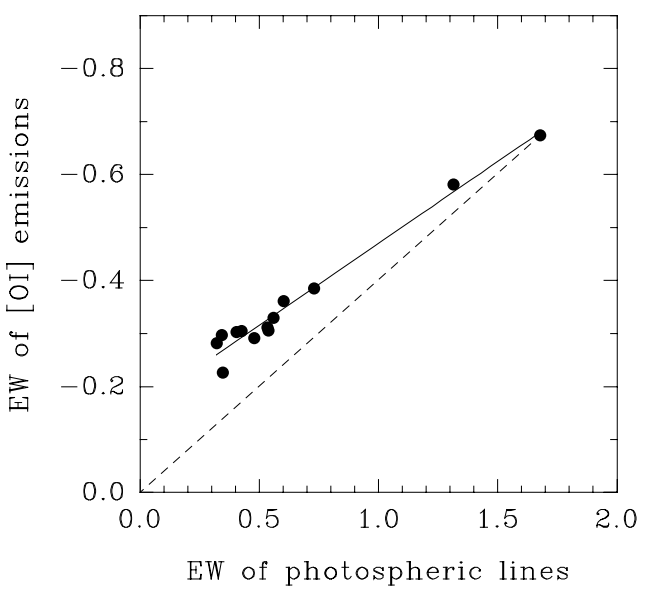

Fig. 7. Correlated variations of $E W \mathrm{~s}$ of forbidden emission lines (sum of [O I ] $\lambda$ 5577, $\lambda 6300$ and $\lambda 6363 \AA$ ) and photospheric lines (sum of 17 lines within 5580-6500 ̊). Dashed line shows relation expected in case of only continuum variations.

DR Tau $E W$ s of about 90 photospheric lines of different elements in the spectral region 5580-6720 A. We then measured the same lines in the template spectrum. For each spectral line we calculated the ratio of $E W \mathrm{~s}$ in DR Tau and in the template spectrum. Identical measurements were performed on the lowveiling spectrum of DR Tau.

If the veiling were only caused by a featureless continuous veiling component, all lines would be reduced by the same factor. However, the ratio of $E W$ s depends on line strength: stronger lines are veiled more (see Fig. 8). The effect as such has been recognized before (e.g. Basri \& Batalha 1990, and references therein) but is here demonstrated in a graphical form. We obtained the same result when we used a synthetic template spectrum instead of the K7V data, and the effect of the differential veiling persists when temperature and gravity of the template vary within probable errors in $T_{\text {eff }}( \pm 200 \mathrm{~K})$ and $\log g( \pm 0.5)$. Note that the scatter of points in Fig. 8 is solely owing to uncertainties in the $E W$ measurements from the spectra of DR Tau and the template. From the weakest lines $(E W \leq 0.05 \AA)$ in Fig. 8 we estimate VF $\sim 0-0.5$ for the low-veiling spectrum, and VF $\sim 3-4$ for the averaged spectrum. These numbers represent upper levels of the veiling continuum in the two spectra. The increasing excess of veiling towards stronger lines $(E W>0.1 \AA)$ should be attributed to chromospheric line emission. The VF values given in Table 1 and shown in Fig. 6 are derived from relatively strong photospheric lines $(E W \approx 0.1 \AA$ in the $\mathrm{K} 7 \mathrm{~V}$ template), because weaker lines are not always measurable in the individual spectra of DR Tau.

The third method uses the colourimetric characteristic of a star with a hot spot (Petrov \& Kozack 2007). According to standard magnetospheric accretion models the continuum veiling originates from the emission of a hot spot located below the accretion stream. The expected effective temperature of the hot spot is $6000-8000 \mathrm{~K}$ with a filling factor within $0.1-1 \%$ of the stellar surface area (Calvet \& Gullbring 1998). It is believed that stochastic events of accretion cause the irregular night-to-night variability of the brightness and colours of cTTS.

The expected colours of a K7 star with a hot photospheric spot can be calculated as a function of spot size and temperature. These two parameters define the veiling factor VF, and hence the calculated colours. This means that, for instance, changes in $B-V$ and $V-R$ are directly related to the degree of veiling. 


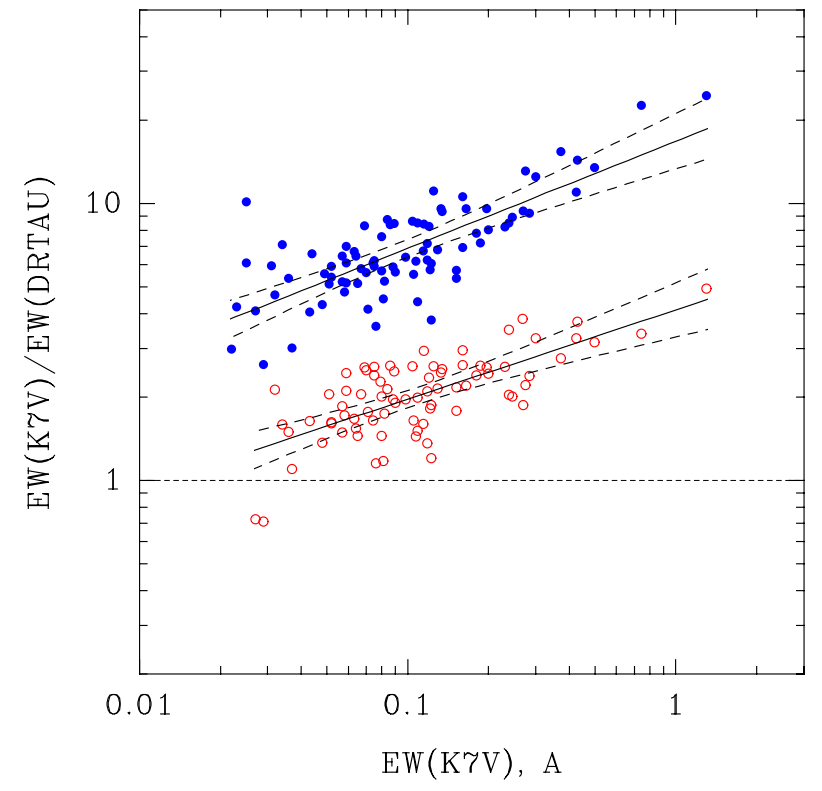

Fig. 8. Ratio of $E W \mathrm{~s}$ of photospheric lines of DR Tau and the observed K7V template HD 28343 as a function of line strength. Filled circles averaged spectrum of DR Tau. Open circles - low-veiling spectrum of DR Tau. Regression lines are shown for both sequences. The $99 \%$ confidence intervals are outlined by dashed curves. The scatter of points around the regression lines is caused by errors of measurements of $E W \mathrm{~s}$. The horizontal dashed line corresponds to zero veiling.

In order to analyse the colour changes in DR Tau we made use of the photometric catalogue by Grankin et al. (2007), which includes about 20 years of observations of TTS. For DR Tau there are over 600 observations in 18 seasons from 1986 to 2003.

The left panel of Fig. 9 shows colour-colour diagrams with the main sequence from A0 to M6, as well as the observed colours of DR Tau. The two solid bow-shaped curves are sequences of the "star+spot" colours, corresponding to two different colour temperatures of the hot spot, approximately those of F0 and A0 stars. Along these curves, the spot radius varies, thus changing the filling factor and, correspondingly, the veiling factor VF. The loci of VF =1, 2, and 4 are marked in the figure. The observed colours deviate from the model grids, but corrections for interstellar reddening must be made.

The right panel of Fig. 9 shows the same diagram with the colours of DR Tau corrected for an interstellar reddening $A_{V}=$ 1 . 0 . This correction brings the cluster of points to the sequence of the "star + spot" colours. The cluster of points is centred at the position of $\mathrm{VF} \approx 1.0$, with a spread from $\mathrm{VF}=0.5$ to $\mathrm{VF}=2$. Some scatter towards bluer $B-V$ colours (about 0.2 ) may be caused by contribution of emission lines to the $B$ pass band.

Apparently, the hot region is permanently present on the visible surface of the star, but does not contribute to the veiling by more than $\mathrm{VF} \approx 2.0$. This corresponds to a brightness variability in the $V$ band within $\approx 1$. 2 . Because the observed veiling of DR Tau reaches much larger values, typically 3 to 10 , the rest of the veiling effect must be caused by the line emission filling-in the photospheric lines. Generally, if the effect of the line emission reduces the photospheric line depth by a factor of $\mathrm{VF}_{\text {line }}$ and, in addition, the non-photospheric continuum reduces the line depth by a factor of $\mathrm{VF}_{\text {cont }}$, the resulting veiling $\mathrm{VF}$ is defined by the relation

$(\mathrm{VF}+1)=\left(\mathrm{VF}_{\text {line }}+1\right) \cdot\left(\mathrm{VF}_{\text {cont }}+1\right)$.
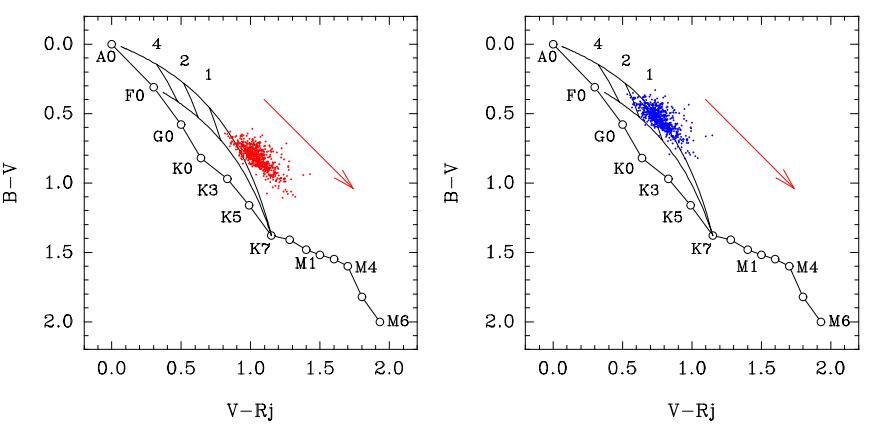

Fig. 9. Two-colour diagrams with a grid of "star + spot" models, shown with the bow-shaped curves. The numbers along the curves represent the amount of veiling in each model. In the left panel, the cluster of dots are the observed colours of DR Tau. In the right panel, a correction for interstellar reddening $A_{V}=1 . \mathrm{m} 0$ has been applied. The slope of the interstellar reddening is indicated with an arrow.

This is a steep relation. For instance, with $\mathrm{VF}_{\text {line }}=\mathrm{VF}_{\text {cont }}=2.0$, the resulting veiling is $\mathrm{VF}=8$. This explains the extremely high veiling $(\mathrm{VF}>10)$ observed sometimes in spectra of cTTS. It also means that the accretion rate derived on the assumption that the observed veiling is caused by an additional continuum may be overestimated by a considerable factor, as was also concluded earlier by Gahm et al. (2008) for other cTTS.

Note that the temperature used in this method is a colour temperature in the spectral range covering the $B V R$ pass bands, and may differ from the effective temperature. The continuum radiation from a hot spot on a cTTS is composed of an optically thick continuum from the heated photosphere and an optically thin Paschen continuum from the pre- and post-shocked gas (Gullbring et al. 2000).

We conclude that all three methods described above show that the veiling continuum in DR Tau is relatively small compared to the values derived directly from measurements of $E W \mathrm{~s}$ of the photospheric lines, indicating that the excess of the observed veiling is caused by the chromospheric line emission.

\subsection{Anti-phase radial velocity variations}

Stellar radial velocity (RV), as measured from positions of photospheric lines, can vary on time scales of days either because of orbital motions in a close binary system or because of surface brightness inhomogeneities (cool/hot spots). In the latter case variations in RV must be within the observed $v \sin i$ value.

Stellar RVs of DR Tau were measured by cross-correlation with the spectrum of the template HD 28343 in the region 5560-6670 $\AA$, with the emission lines masked out. The radial velocity of the template was determined by cross-correlation with the synthetic spectrum $\left(T_{\text {eff }}=4000 \mathrm{~K}, \log g=4.5\right)$ resulting in $\mathrm{RV}=-35.20 \pm 0.04 \mathrm{~km} \mathrm{~s}^{-1}$. The results of the RV measurements are given in Table 2.

We then selected a group of unblended emission lines of the Fe I multiplet 15, within 5371-5456 $\AA$, as representative of the chromospheric emission (most lines are present in Fig. 1). The profile of each line was decomposed into a broad and narrow component, and the position of the narrow component was determined by a Gaussian fit. The average RVs of the Fe I emission lines are also given in Table 2. The two blanks in the column of $\mathrm{RV}_{\mathrm{em}}$ correspond to dates when the star was at a state of low veiling and the Fe I emission was too weak to be used for RV measurements. The RVs show night-to-night variations with a semiamplitude of about $1 \mathrm{~km} \mathrm{~s}^{-1}$ for both the photospheric and the 
Table 2. Radial velocities of photospheric and chromospheric lines in DR Tau.

\begin{tabular}{ccc}
\hline \hline HJD-245 .. & $\begin{array}{c}\mathrm{RV}_{\text {phot }} \\
\left(\mathrm{km} \mathrm{s}^{-1}\right)\end{array}$ & $\begin{array}{c}\mathrm{RV}_{\mathrm{em}} \\
\left(\mathrm{km} \mathrm{s}^{-1}\right)\end{array}$ \\
\hline 4101.4056 & $21.53 \pm 0.35$ & $22.12 \pm 0.34$ \\
4103.5857 & $23.08 \pm 0.33$ & $(\ldots)$ \\
4104.4278 & $22.97 \pm 0.15$ & $(\ldots)$ \\
4105.6235 & $23.14 \pm 0.37$ & $21.62 \pm 0.23$ \\
5192.5623 & $23.36 \pm 0.18$ & $22.19 \pm 0.17$ \\
5193.4952 & $23.08 \pm 0.36$ & $23.39 \pm 0.31$ \\
5194.4984 & $22.78 \pm 0.29$ & $23.24 \pm 0.24$ \\
5195.4934 & $22.98 \pm 0.19$ & $22.24 \pm 0.18$ \\
5196.4939 & $23.78 \pm 0.25$ & $21.70 \pm 0.22$ \\
5197.5002 & $23.31 \pm 0.23$ & $22.28 \pm 0.28$ \\
5198.5539 & $22.69 \pm 0.15$ & $24.00 \pm 0.19$ \\
5199.5762 & $22.96 \pm 0.47$ & $22.36 \pm 0.19$ \\
5201.4676 & $23.30 \pm 0.21$ & $22.06 \pm 0.32$ \\
\hline
\end{tabular}

Table 3. Mean RV and range of RV variations.

\begin{tabular}{lcc}
\hline \hline Line & $\begin{array}{c}\text { Mean RV } \\
\left(\mathrm{km} \mathrm{s}^{-1}\right)\end{array}$ & $\begin{array}{c}\text { Semi-amplitude } \\
\left(\mathrm{km} \mathrm{s}^{-1}\right)\end{array}$ \\
\hline Photospheric lines & $23.0 \pm 0.2$ & $1.1 \pm 0.2$ \\
\hline Narrow component: $\mathrm{Fe} \mathrm{I}_{\mathrm{I}}$ & $22.5 \pm 0.2$ & $1.1 \pm 0.2$ \\
$\mathrm{Fe}_{\text {II }}$ & $23.4 \pm 0.3$ & $1.5 \pm 0.2$ \\
$\mathrm{He}_{\text {I }}$ & $26.9 \pm 0.9$ & $1.1 \pm 0.3$ \\
$\mathrm{He}_{\text {II }}$ & $36.1 \pm 0.5$ & $2.5 \pm 0.7$ \\
\hline Broad component: Fe I, Fe II & $24.3 \pm 1.0$ & $5.0 \pm 1.5$ \\
$\mathrm{He}_{\text {I }}$ & $22.9 \pm 1.0$ & $8 \pm 2$ \\
\hline
\end{tabular}

emission lines, which is well within the $v \sin i$ value. The main result is that the $R V$ variations of the photospheric lines and of the narrow component of the Fe I emissions are in anti-phase (see Fig. 10). Similar variations were first reported for RW Aur by Petrov et al. (2001).

The broad components show periodic shifts in central velocity with time. However, the measurement accuracy of the position of a broad component using a Gaussian fit is much lower than for the narrow components. The RV amplitudes of broad components were measured by means of cross-correlation of each spectrum with the average spectrum of DR Tau with the narrow components masked out. The amplitudes of the broad components turned out to be larger than those of the narrow components, which is consistent with an origin of the broad components well above the stellar surface.

Table 3 summarises measurements of RVs in different groups of lines, which are arranged in order of increasing RV amplitude. The mean stellar velocity is $+23 \mathrm{~km} \mathrm{~s}^{-1}$. Note that the mean RV is more positive for the "high-temperature" lines, up to $+36 \mathrm{~km} \mathrm{~s}^{-1}$ for the narrow He II component.

Our search for radial velocity changes was then extended to the other cTTS included in the present study. As shown in Fig. 11, all programme stars show the same type of anti-phase variations as observed for DR Tau. Furthermore, narrow emission lines from ions like $\mathrm{He}$ I formed at higher temperatures are also red-shifted by several $\mathrm{km} \mathrm{s}^{-1}$ with respect to the stellar velocity. Therefore these phenomena, indicating infall to regions at the stellar surface that are offset relative to the rotational axis, appear to be a universal property of active cTTS.
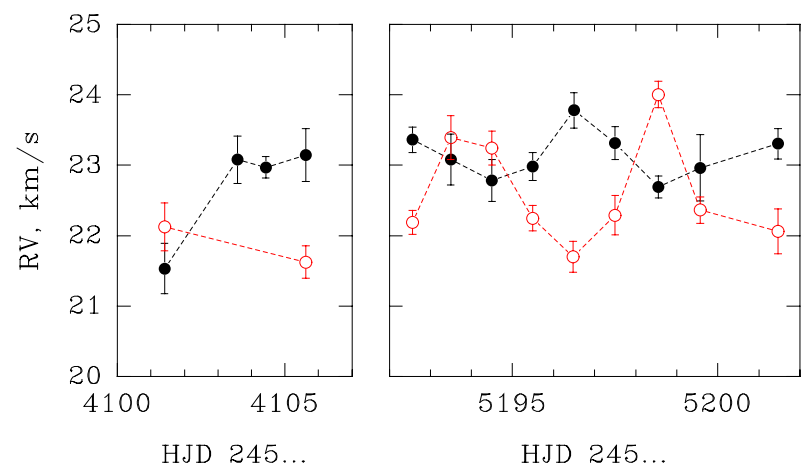

Fig. 10. Anti-phase RV-variations of photospheric (filled circles) and chromospheric (open circles) lines in DR Tau.
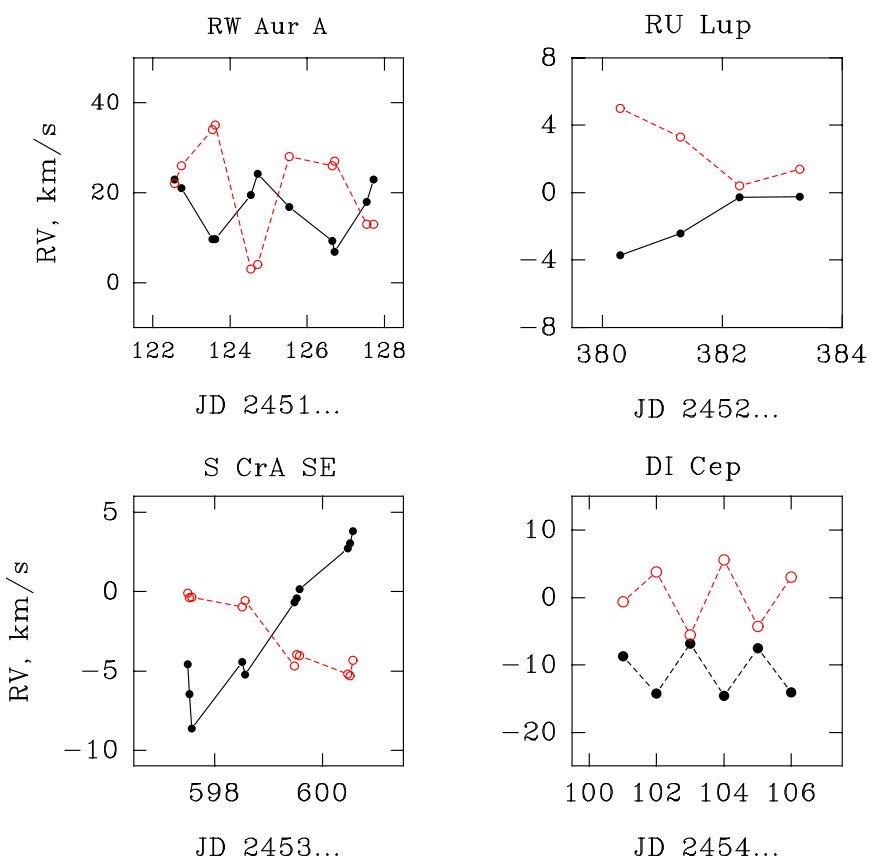

Fig. 11. Anti-phase RV-variations of photospheric (filled circles) and chromospheric (open circles) lines in four cTTS. Narrow emission lines of $\mathrm{Fe}_{\mathrm{I}}$ were measured in RW Aur and S CrA SE, and of $\mathrm{He}_{\mathrm{I}}$ in RU Lup and DI Cep. Note the positive shift in velocity of the He I emission.

\section{Discussion}

\subsection{Modelling the RV variations}

Cross-correlation of DR Tau spectra with the synthetic spectrum in the region 6000-6040 $\AA$ reveals that the bisector of the crosscorrelation function (as described in Dall et al. 2006) correlates with the stellar radial velocity. This excludes the case of a binary motion and suggests that RV variations are caused by distortions in the photospheric line profiles. The same effect was found earlier for RU Lup by Stempels et al. (2007).

The anti-phase RV variations of the photospheric and emission lines suggest that the line emission is confined to a certain area on the stellar surface, which is not aligned with the axis of rotation, resulting in periodic shifts in $\mathrm{RV}$ as the star rotates. The velocity curves in Fig. 10 have minima and maxima separated by about 4.5 days, which is similar to the photometric and spectroscopic periods (4.5-5.0 days) reported above, and which have been linked to rotational modulation.

The effect of an "emission spot" resulting in the anti-phase RV variations can be illustrated with a simple model. We 
Table 4. Stellar and chromospheric spot model parameters.

\begin{tabular}{lccccc}
\hline \hline \multicolumn{5}{c}{ Stellar parameters } \\
$T_{\text {eff }}$ & $\log g$ & $L_{*}$ & $R_{*}$ & $v \sin i$ & $i$ \\
$(\mathrm{~K})$ & & $\left(L_{\odot}\right)$ & $\left(R_{\odot}\right)$ & $\left(\mathrm{km} \mathrm{s}^{-1}\right)$ & $(\mathrm{deg})$ \\
\hline 4100 & 3.7 & 0.52 & 1.46 & 5.0 & 20 \\
\hline \multicolumn{5}{c}{ Chromospheric spot parameters } \\
$r_{\text {spot }}$ & $\phi$ \\
$(\mathrm{deg})$ & $(\mathrm{deg})$ & & & \\
\hline 14 & 76 & & \\
\hline
\end{tabular}

generated synthetic spectra of a single rotating star plus an area emitting the narrow emission lines under the assumption that this area has the form of a circular spot. The input parameters for the model are: projected rotational velocity $(v \sin i)$, inclination $(i)$ of the stellar rotational axis to the line of sight, spot latitude $(\phi)$, spot radius $\left(r_{\text {spot }}\right)$, local spectra of the photosphere and the chromospheric spot, and the limb darkening coefficient $(\mu)$. The spectrum was integrated over the visible stellar surface at different phases of stellar rotation. We then determined the radial velocities of the photospheric lines $R V_{\text {phot }}$ and the emission lines $\mathrm{RV}_{\mathrm{em}}$ as a function of phase.

In this model most of the input parameters are restricted by observations. From $v \sin i=5 \pm 1 \mathrm{~km} \mathrm{~s}^{-1}$, rotational period 4.5-5.0 days and $R_{*}=1.46 R_{\odot}$, we obtain the inclination angle $i=20^{\circ} \pm 4^{\circ}$, i.e. the star is seen close to pole-on. The inclination is not very sensitive to the adopted $A_{V}$, because the slopes of lines of equal radii in the $V$ versus $(V-R)$ diagram and reddening are the same.

The spot latitude $(\phi)$ on the stellar surface can be estimated from the amplitude $\Delta \mathrm{RV}$ of the radial velocity variations of the Fe I emission lines: $\Delta \mathrm{RV}=v \sin i \cdot \cos (\phi)$. With $v \sin i=5.0 \pm$ $1.0 \mathrm{~km} \mathrm{~s}^{-1}$ and $\Delta \mathrm{RV}=1.15 \pm 0.20 \mathrm{~km} \mathrm{~s}^{-1}$ we get $\phi=76^{\circ} \pm 4^{\circ}$.

A synthetic spectrum with $T_{\text {eff }}=4100 \mathrm{~K}$ and $\log g=3.7$ was taken as the local spectrum of the undisturbed photosphere surrounding the spot. Within the spot area the local spectrum was assumed to be a sum of the photospheric spectrum and a line emision spectrum with similar line widths and line ratios. The overall emission line strength was a free parameter. The limb darkening coefficient was taken as $\mu=0.8$ (Al-Naimi 1978). Table 4 summarises the model parameters.

As shown in Fig. 12 the model can reproduce the observed anti-phase RV-variations very well. The emission line strengths were tuned to obtain the best fit to the observed RV amplitudes. Equal RV amplitudes of emission and absorption lines occur when the latter is about halfway filled with emission (i.e. $\mathrm{VF}_{\text {line }} \approx 1$ ). A spot radius of $r_{\text {spot }}=14^{\circ}$ (filling factor $=0.015$ ) adopted in this model is about the same as the typical radius of the accretion stream in the 3D simulations of magnetospheric accretion by Romanova et al. (2003). The model also reproduces the redshifted velocity offset of the He lines, provided that this emission is formed in infalling gas, with a velocity vector directed towards the star.

In some other cTTS, the Zeeman-Doppler imaging technique has been used to reconstruct the large-scale magnetic topology and to outline Ca II line emission from the shocked gas near the stellar surface: V2129 Oph (Donati et al. 2007, and 2011), BP Tau (Donati et al. 2008), CV Cha and CR Cha (Hussain et al. 2009), and AA Tau (Donati et al. 2010). While the recovered structures are sometimes complex, it is not uncommon to see hot spots, as indicated by the enhanced $\mathrm{Ca}$ II emission. The adopted size and derived location relative to the rotational axis for our
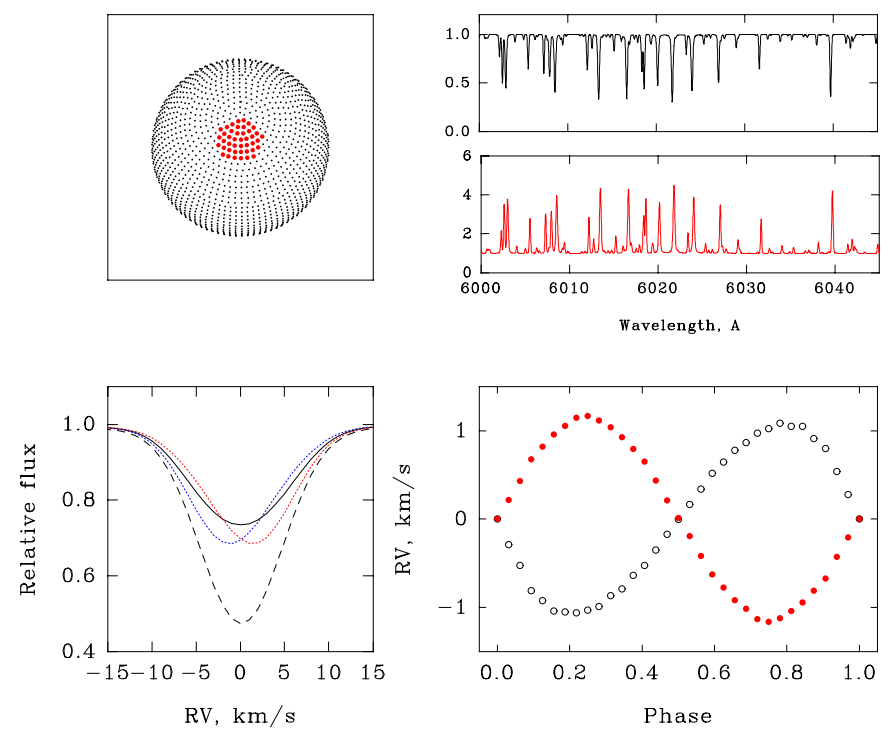

Fig. 12. Upper panels: location of the chromospheric spot and the local spectra corresponding to the undisturbed photosphere (absorption) and to the spot (emission). Lower panels: left - profiles of the Ca I $\lambda 6039 \AA$ line, corresponding to phases 0.0 (spot facing the observer, solid line), 0.22 and 0.78 (maximal blue- and red-shifted absorption, dotted lines). Dashed profile: a normal photosphere without spot; right - RV-variations of absorption lines (open circles) and emission lines (filled circle).

Fe I emitting area in DR Tau resembles the spots of Ca II emission found in V2129 Oph and BP Tau.

It is worthwhile to note that the distortions of a photospheric line profile caused by an emission core (Fig. 12) are very similar to those caused by a dark spot. The two effects can be distinguished with the help of photometry made simultaneously with spectropolarimetry. Although we cannot discard a contribution from cool spots in the observed RV-variations of photospheric lines in DR Tau, both the differential veiling and the anti-phase $\mathrm{RV}$-variations indicate that these are primarily caused by line emission. The presence of narrow emission lines inside photospheric lines is particularly evident in S CrA SE (Gahm et al. 2008).

\subsection{Accretion powered emission}

A direct signature of infall are the inverse P Cyg (IPC) absorption components. In our series of spectra of DR Tau, IPC are present in the strongest Fe II lines (multiplet 42), in the $\mathrm{Na}$ I $\mathrm{D}$ lines, in the higher Balmer lines and, less evident, in the $\mathrm{He}_{\mathrm{I}}$ line at $5876 \AA$ (Fig. 2). In the low-veiling spectrum most emission lines have faded out, and only the strongest emission lines are present. On this occasion the IPC profiles have also disappeared. During this episode of relatively weak accretion both the veiling continuum and the chromospheric emission are considerably reduced. The upper limit to the veiling continuum that day was only $\sim 0.5$, as is evident from Fig. 8 . The area of enhanced chromospheric emission in DR Tau is permanently present, but varies in strength, and is probably coupled to a variable amount of mass accretion.

We can roughly estimate the expected accretion luminosity for the low-veiling spectrum using the relation between $\mathrm{H} \alpha$ emission line flux and accretion luminosity by Fang et al. (2009). Our spectra are not flux-calibrated, but for the lowveiling spectrum we can assume that DR Tau was close to its 

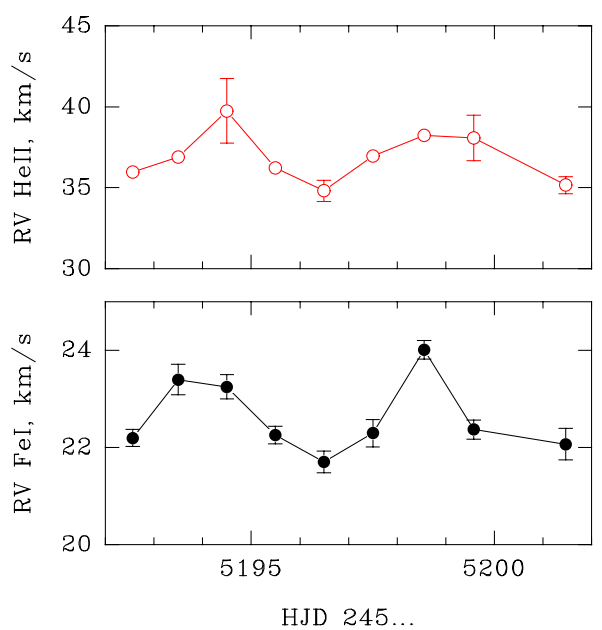

Fig. 13. Correlated variations of $\mathrm{RV}$ of the narrow emission of $\mathrm{Fe}_{\mathrm{I}}$ and He II.

minimum brightness (see Sect. 3.2). In the low-veiling spectrum, $E W(\mathrm{H} \alpha)=83.5 \AA$. For a K7V star with $L_{*}=0.5 L_{\odot}$, this $E W$ corresponds to $\log \left(L_{\mathrm{H} \alpha} / L_{\odot}\right)=-2.53$. Using the relation by Fang et al. (2009), we obtain $\log \left(L_{\text {accr }} / L_{\odot}\right)=-0.89 \pm 0.25$, or $L_{\text {accr }} / L_{*} \approx 0.26$, which is compatible with an observed veiling $<0.5$ in the $V$ band.

To explore further whether the narrow $\mathrm{Fe}_{\mathrm{I}}$ emission is related to the hot accretion spot, we followed radial velocity variations of the He II $\lambda 4686 \AA$ emission. In standard dipole magnetospheric accretion models $\mathrm{He}$ is ionised by the $\mathrm{X}$-ray radiation from the shock. Figure 13 shows that the RV of the narrow Fe I emission lines vary in phase with the narrow He II emission line. This strongly suggests that the area of chromospheric emission is located close to the hot region at the footprint of the accretion stream (see also discussions by Batalha et al. 1996; and Beristain et al. 1998). Moreover, the He II line is clearly red-shifted relative to other narrow lines. As discussed by e.g. Lamzin (1998), these "high-temperature" lines are formed in the post-shock region.

Our observations thus demonstrate that there is a close relation between the appearance of enhanced chromospheric emission and accretion in classical T Tauri stars. The narrow emission lines of e.g. Fe I appears over an area at the stellar surface that is associated with the footprints of the magnetic funnels, and that is offset from the pole of rotation.

In standard dipole magnetospheric models the accretion spot may take the form of a ring, which becomes distorted when the magnetic and rotational axes are not aligned. However, recent models assume much more complex magnetic field configurations (e.g. Mohanty \& Shu 2008). The 3D simulations of magnetically channelled accretion by e.g. Romanova et al. (2004, 2011) and Long et al. (2011) reflect the complex magnetic structures found from Doppler imaging of TTS.

In the general concept of magnetically channeled accretion, the accretion spot on a cTTS determines the observed brightness of the star, the veiling of photospheric lines and the strength of certain emission lines, and all three parameters must vary in correlation. However, observations do not always support this prediction (see e.g. Petrov et al. 2001). In order to resolve this discrepancy, we suggest that the area of enhanced chromospheric emission is much more extended than the size of the hot accretion spot. In the models by Romanova et al., less dense and non-uniform gas surrounds the main accretion stream and falls onto a much larger area than what is covered by the main stream, up to $16 \%$ of the stellar surface. A possibility is that our observed enhanced chromospheric emission is excited by such a milder accretion flow and therefore distributed over a similar area surrounding the smaller hot spot. One can assume that the accompanying shocks and related phenomena are less pronounced than at the main footprints, and that little or no continuous excess emission is released. In this scenario, a tight correlation between the brightness, veiling and emission line strength is not expected.

X-ray observations of cTTS show that besides very powerful coronal emission there is as a rule one component of soft X-rays that can be associated with the hot spot (see e.g. Güdel \& Telleschi 2007; and Argiroffi et al. 2009, and references therein). Recently, Brickhouse et al. (2010) presented X-ray spectroscopy of the cTTS TW Hya. A low-temperature component $(T \sim 2.5 \mathrm{MK})$ could be identified with the accretion shock, as depicted in the standard accretion models, and a high-temperature component was ascribed to coronal emission at $T \sim 10 \mathrm{MK}$. In addition, the authors discuss a third component, represented by low-density lines of e.g. O VII formed at $T \sim 1.75 \mathrm{MK}$, which fills a great volume outside the post-shock region. Brickhouse et al. speculate that violent mass outflows and shocks may propagate from the base of the accretion shock (see also Orlando et al. 2010), and that the photospheric surroundings are heated, which supplies ionised gas to accretionfed magnetic structures, such as magnetic loops. In addition, one can expect that the surface layers surrounding the hot spot are excited by UV-light directly from the hot spot.

Regardless of the dominating cause of the enhanced chromospheric emission one would expect that the atmospheric structure adjacent to the hot spot is affected. In some early attempts to explain line emission and veiling in cTTS, Herbig (1970) and Cram $(1979,1980)$ introduced the concept of a "deep chromosphere", where the temperature minimum occurs at deeper layers in the atmosphere, thus resulting in the appearence of stronger emisson spectrum. We speculate that our area of enhanced emission surrounding the hot spot could be similar to a deep chromosphere with a temperature minimum much deeper in the atmosphere than in a normal dwarf, but still above the photospheric continuum. In any case, the chromospheric emission in cTTS appears to be related not only to solar-like magnetic activity, but is to a considerable extent powered by accretion processes.

\section{Conclusions}

We have studied periodic changes in photospheric lines and narrow components of emission lines in DR Tauri and other classical T Tauri stars, and investigated the nature of the dilution of photospheric lines, the so-called veiling. In particular we note:

- In the course of our observations the amount of veiling in DR Tau varies from practically nothing to factors of more than 10 times the stellar continuum intensity. We have developed three methods to distinguish the contribution from line emission and continuous excess emission to the veiling. We conclude that narrow emission lines fill in the photospheric absorption lines, and that this effect is more pronounced when the veiling is strong. Therefore, estimates of mass accretion rates based on the assumption of a continuous veiling will be overestimated for cTTS with strong emission line spectra.

- Radial velocities of photospheric and narrow chromospheric lines vary in anti-phase in all cTTS studied here. The mean velocity of the narrow lines are at rest relative to the stellar 
radial velocity. The velocity amplitudes are small. We have modelled these variations and infer that the emission lines are formed in an area at the stellar surface that is offset from the rotational axis. These properties appears to be a common phenomenon in cTTS.

- The narrow emission line of He II $\lambda 4686 \AA$ in DR Tau varies in phase with the narrow chromospheric components, but is systematically red-shifted by $\sim 10 \mathrm{~km} \mathrm{~s}^{-1}$, which is consistent with the origin of this line in decelerated post-shock gas. We conclude that the area of enhanced chromospheric emission is directly related to the hot spot area, and that chromospheric emission is induced by accretion. In accordance with this, we demonstrate that a sudden disappearance of inverse $\mathrm{P}$ Cygni profles in strong lines coincided with a fading of both the veiling and the strength of chromospheric lines.

We suggest that the area of enhanced chromospheric emission comes from an extended region at the stellar surface surrounding the hot spot. The physical mechanism that triggers the emission can be related to current ideas that differ from the standard model in that accretion is more wide-spread, or emanates from injection of mass and energy from the shocked region under the main accretion stream. As a result the photosphere surrounding the hot spot is heated, which leads to a modified atmospheric structure, which in turn produces the enhanced emission in narrow emission lines responsible for the extra component in the veiling through line-filling of photospheric absorption lines.

Acknowledgements. This work was supported by the INTAS grant 03-51-6311. H.C.S. acknowledges grant 621-2009-4153 of the Swedish Research Council.

\section{References}

Aiad, A., Appenzeller, I., Bertout, C., et al. 1984, A\&A, 130, 67 Alencar, S. H. P., Johns-Krull, C. M., \& Basri, G. 2001, ApJ, 122, 3335 Al-Naimi, H. M. 1978, Ap\&SS, 53, 181

Appenzeller, I., Reitermann, A., \& Stahl, O. 1988, PASP, 100, 815 Ardila, D. A., Basri, G., Walter, F. M., Valenti, J. A., \& Johns-Krull, C. M. 2005, ApJ, 567, 1013

Argiroffi, C., Maggio, A., Peres, G., et al. 2009, A\&A, 507, 939

Artemenko, S. A., Grankin, K. N., \& Petrov, P. P. 2010, Astron. Rep., 54, 163

Basri, G., \& Batalha, C. 1990, ApJ, 363, 654

Batalha, C. C., Stout-Batalha, N. M., Basri, G., \& Terra, M. A. O. 1996, ApJS, 103,211

Berdyugina, S. V. 1991, Bull. Crimean Astrophys. Obs., 83, 89

Beristain, G., Edwards, S., \& Kwan, J. 1998, ApJ, 499, 828

Beristain, G., Edwards, S., \& Kwan, J. 2001, ApJ, 551, 1037

Bertout, C. 1984, Rep. Prog. Phys., 47, 111

Bertout, C., \& Basri, G. 1988, ApJ, 330, 350

Bertout, C., \& Genova, F. 2006, A\&A, 460, 499

Bertout, C., Krautter, J., Moellenhoff, C., \& Wolf, B. 1977, A\&A, 61, 73

Bouvier, J., Cabrit, S., Fernandez, M., Martin, E. L., \& Matthews, J. M. 1993, A\&A, 272, 176

Bouvier, J., Covino, E., Kovo, O., et al. 1995, A\&A, 299, 89

Bouvier, J., Alencar, S. H. P., Harries, T. J., Johns-Krull, C. M., \& Romanova,

M. M. 2007, in Protostars and Planets V., ed. B. Reipurth, D. Jewitt, \& K. Keil, 479

Brickhouse, N. S., Cranmer, S. R., Dupree, A. K., Luna, G. J. M., \& Wolk, S. 2010, ApJ, 710, 1835

Calvet, N., \& Gullbring, E. 1998, ApJ, 509, 802

Carmona, A., Van Den Ancker, M. E., \& Henning, T. 2007, A\&A, 464, 687
Chavarria-K., C. 1979, A\&A, 79, L18

Cram, L. E. 1979, ApJ, 234, 949

Cram, L. E., Giampapa, M. S., \& Imhoff, C. L. 1980, ApJ, 238, 905

Dall, T. H., Santos, N. C., Arentoft, T., Bedding, T. R., \& Kjeldsen, H. 2006, A\&A, 454, 341

Donati, J.-F., Jardine, M. M., Gregory, S. G., et al. 2007, MNRAS, 380, 1297

Donati, J.-F., Jardine, M. M., Gregory, S. G., et al. 2008, MNRAS, 386, 1234

Donati, J.-F., Skelly, M. B., Bouvier, J., et al. 2010, MNRAS, 409, 1347

Donati, J.-F., Bouvier, J., Walter, F. M., et al. 2011, MNRAS, 412, 2454

Edwards, S., Fischer, W., Kwan, J., Hillenbrand, L., \& Dupree, A. K. 2003, ApJ, 599, L41

Edwards, S., Fischer, W., Hillenbrand, L., \& Kwan, J. 2006, ApJ, 646, 319

Fang, M., van Boekel, R., Wang, W., et al. 2009, A\&A, 504, 461

Fischer, W., Kwan, J., Edwards, S., \& Hillenbrand, L. 2008, ApJ, 687, 1117

Frandsen, S., \& Lindberg, B. 2000, in The Third MONS Workshop: Science Preparation and Target Selection, ed. T. Teixeira, \& T. Bedding, 163

Gahm, G. F., Walter, F. M., Stempels, H. C., Petrov, P. P., \& Herczeg, G. J. 2008, A\&A, 482, L35

Gameiro, J. F., Folha, D. F. M., \& Petrov, P. P. 2006, A\&A, 445, 323

Grankin, K. N., Melnikov, S. Yu., Bouvier, J., Herbst, W., \& Shevchenko, V. S. 2007, A\&A, 461, 183

Guenther, E., \& Hessman, F. V. 1993, A\&A, 268, 192

Gullbring, E., Hartmann, L., Briceño, C., \& Calvet, N. 1998, ApJ, 492, 323

Gullbring, E., Calvet, N., Muzerolle, J., \& Hartmann, L. 2000, ApJ, 544, 927

Güdel, M., \& Telleschi, A. 2007, A\&A, 474, L25

Hartigan, P., Kenyon, S. J., Hartmann, L., et al. 1991, ApJ, 382, 617

Hartigan, P., Edwards, S., \& Ghandour, L. 1995, ApJ, 452, 736

Herbig, G. H. 1970, Mem. Soc. Roy. Sci. Liege, 5th Ser., 19, 13

Hessman, F. V., \& Guenther, E. 1997, A\&A, 321, 497

Hussain, G. A. J., Collier Cameron, A., Jardine, M. M., et al. 2009, MNRAS, 398,189

Johns, C. M., \& Basri, G. 1995, AJ, 109, 2800

Johns-Krull, C. M., \& Gafford, A. D. 2002, ApJ, 573, 685

Kenyon, S. J., Hartmann, L., Hewett R., et al. 1994, AJ, 107, 2153

Krautter, J., \& Bastian, U. 1980, A\&A, 88, L6

Kravtsova, A. S., \& Lamzin, S. A. 1996, Astron. Lett., 28, 835

Kwan, J., Edwards, S., \& Fischer, W. 2007, ApJ, 657, 897

Kupka, F., Ryabchikova, T. A., Piskunov, N. E., Stempels, H. C., \& Weiss, W. W. 2000, Balt. Astron., 9, 590

Lamzin, S. A. 1998, Astron. Rep., 42, 322

Lamzin, S. A., Bisnovatyi-Kogan, G. S., Errico, L., et al. 1996, A\&A, 306, 877

Long, M., Romanova, M. M., Kulkarni, A. K., \& Donati, J.-F. 2011, MNRAS, 413, 1061

Lorenzetti, D., Larionov, V. M., Giannini, T., et al. 2009, ApJ, 693, 1056

Mohanty, S., \& Shu, F. H. 2008, ApJ, 687, 1323

Mundt, R. 1984, ApJ, 280, 749

Muzerolle, J., Hartmann, L., \& Calvet, N. 1998a, AJ, 116, 455

Muzerolle, J., Hartmann, L., \& Calvet, N. 1998b, ApJ, 492, 743

Orlando, S., Sacco, G. G., Argiroffi, C., et al. 2010, A\&A, 510, A71

Percy, J. R., Grinko, S., Seneviratne, R., \& Hebst, W. 2010, PASP, 122, 753

Petrov, P. P. 2003, Astrophysics (English translation of Astrofizika), 46, 4

Petrov, P. P., \& Kozack, B. S. 2007, Astron. Rep., 51, 500

Petrov, P. P., Gahm, G. F., Gameiro, J. F., et al. 2001, A\&A, 369, 993

Romanova, M. M., Ustyugova, G. V., Koldoba, A. V., Wick, J. V., \& Lovelace, R. V. E. 2003, ApJ, 595, 1009

Romanova, M. M., Ustyugova, G. V., Koldoba, A. V., \& Lovelace, R. V. E. 2004, ApJ, 610, 920

Romanova, M. M., Long, M., Lamb, F. K., Kulkarni, A. K., \& Donati, J.-F. 2011, MNRAS, 411, 915

Richter, M., Basri, G., Perlmutter, S., \& Pennypacker, C. 1992, PASP, 104, 1114 Smith, K. W., Bonnell, I. A., Lewis, G. F., \& Bunclark, P. S. 1997, MNRAS, 289, 151

Smith, K. W., Lewis, G. F., Bonnell, I. A., Bunclark, P. S., \& Emerson, J. P. 1999, MNRAS, 304, 367

Stempels, H. C., \& Piskunov, N. 2002, A\&A, 391, 595

Stempels, H. C., Gahm, G. F., \& Petrov, P. P. 2007, A\&A, 461, 253

Valenti, J. A., Basri, G., \& Johns, C. M. 1993, AJ, 106, 2024 